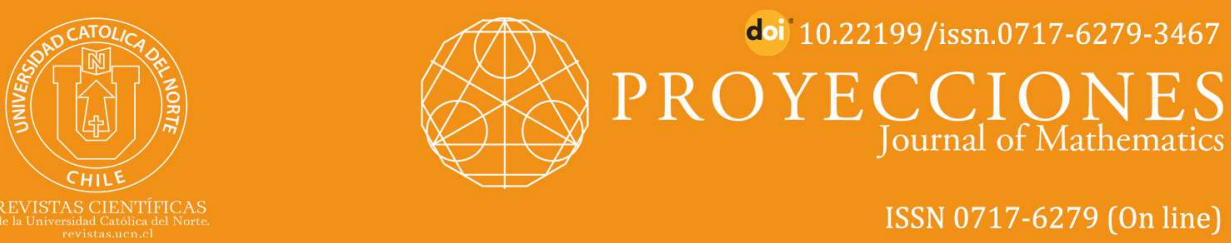

\title{
On graded primary-like submodules of graded modules over graded commutative rings
}

Khaldoun Al-Zoubi ${ }^{1 *}$ (D) orcid.org/0000-0001-6082-4480

Mohammed Al-Dolat ${ }^{2}$ @ orcid.org/0000-0003-2738-2072

Jordan University of Science and Technology, Dept. of Mathematics and Statistics, Irbid, Jordan.

1曰kfzoubi@just.edu.jo; ${ }^{2}$ mmaldolat@just.edu.jo

\section{Abstract:}

Let $G$ be a group with identity e. Let $R$ be a $G$-graded commutative ring andM a graded $R$-module. In this paper, we introduce the concept of graded primary-like submodules as a new generalization of graded primary ideals and give some basic results about graded primary-like submodules of graded modules. Special attention has been paid, when graded submodules satisfies the grprimeful property, to and extra properties of these graded submodules.

Keywords: Graded primary ideals; Graded primary-like submodules; Graded prime submodules, gr-primeful property.

MSC (2020): 13A02, 16W50.

\section{Cite this article as (IEEE citation style):}

K. Al-Zoubi and M. Al-Dolat, "On graded primary-like submodules of graded modules over graded commutative rings", Proyecciones (Antofagasta, On line), vol. 40, no. 4, pp. 859-871, 2021, doi: 10.22199/issn.0717-6279-3467

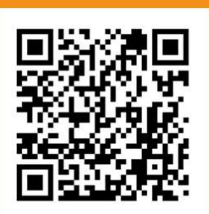

Article copyright: (C) 2021 Khaldoun Al-Zoubi and Mohammed Al-Dolat. This is an open access article distributed under the terms of the Creative Commons License, which permits unrestricted use and distribution provided the original author and source are credited.

*Corresponding author 


\section{Introduction}

Recently, H. F. Moghimi and F. Rashedi, in [17] studied primary-like submodules as a new generalization of primary ideals to modules. Also, the concept of primeful module was introduced and studied by C.P. Lu in [15].

The scope of this paper is devoted to the theory of graded modules over graded commutative rings. One use of rings and modules with gradings is in describing certain topics in algebraic geometry. Here, in particular, we are dealing with graded primary-like submodules.

The concept of graded primary ideal was introduced and studied by M. Refai and K. Al-Zoubi in [22].

In the literature, there are several different generalization of the notion of graded primary ideal to graded module. The concept of graded primary submodule was introduced by S.E. Atani and F. Farzalipour in [11] and studied in $[1,4,13,21]$. Also the the concept of graded prime submodule was introduced by S.E. Atani in [7] and studied in $[2,3,5,6$, $8,9,16,21,24]$.

Here, we introduce the concept of graded primary-like submodule as a new generalization of a graded primary ideal on the one hand and a generalization of a graded prime submodule on other hand.

Our article is organized as follows.

In Section 2 we recall important notions which will be used throughout the paper. In Section 3 we will investigate graded submodules which satisfy the gr-primeful property. In Section 4 we introduce the concept of graded primary-like submodules and give a number of results concerning such modules. For example, we give a characterization of graded primary-like submodules. We also study the behavior of graded primary-like submodules under graded homomorphisms and under localization.

\section{Preliminaries}

Convention. Throughout this paper all rings are commutative with identity and all modules are unitary.

First, we recall some basic properties of graded rings and modules which will be used in the sequel. We refer to [14], [18], [19] and [20] for these basic properties and more information on graded rings and modules.

Let $G$ be a group with identity $e$ and $R$ be a commutative ring with identity $1_{R}$. Then $R$ is a $G$-graded ring if there exist additive subgroups $R_{g}$ of $R$ such that $R=\bigoplus_{g \in G} R_{g}$ and $R_{g} R_{h} \subseteq R_{g h}$ for all $g, h \in G$. The 
elements of $R_{g}$ are called to be homogeneous of degree $g$ where the $R_{g}$ 's are additive subgroups of $R$ indexed by the elements $g \in G$. If $x \in R$, then $x$ can be written uniquely as $\sum_{g \in G} x_{g}$, where $x_{g}$ is the component of $x$ in $R_{g}$. Moreover, $h(R)=\bigcup_{g \in G} R_{g}$. Let $I$ be an ideal of $R$. Then $I$ is called a graded ideal of $(R, G)$ if $I=\bigoplus_{g \in G}\left(I \cap R_{g}\right)$. Thus, if $x \in I$, then $x=\sum_{g \in G} x_{g}$ with $x_{g} \in I$. An ideal of a $G$-graded ring need not be $G$-graded.

Let $R$ be a $G$-graded ring and $M$ an $R$-module. We say that $M$ is a $G$-graded $R$-module (or graded $R$-module) if there exists a family of subgroups $\left\{M_{g}\right\}_{g \in G}$ of $M$ such that $M=g \in G \oplus M_{g}$ (as abelian groups) and $R_{g} M_{h} \subseteq M_{g h}$ for all $g, h \in G$. Here, $R_{g} M_{h}$ denotes the additive subgroup of $M$ consisting of all finite sums of elements $r_{g} s_{h}$ with $r_{g} \in R_{g}$ and $s_{h} \in M_{h}$. Also, we write $h(M)=g \in G \cup M_{g}$ and the elements of $h(M)$ are called to be homogeneous . Let $M=g \in G \bigoplus M_{g}$ be a graded $R$-module and $N$ a submodule of $M$. Then $N$ is called a graded submodule of $M$ if $N=g \in G \oplus N_{g}$ where $N_{g}=N \cap M_{g}$ for $g \in G$. In this case, $N_{g}$ is called the $g$-component of $N$.

Let $R$ be a $G$-graded ring and $S \subseteq h(R)$ be a multiplicatively closed subset of $R$. Then the ring of fraction $S^{-1} R$ is a graded ring which is called the graded ring of fractions. Indeed, $S^{-1} R=g \in G \oplus\left(S^{-1} R\right)_{g}$ where $\left(S^{-1} R\right)_{g}=\left\{r / s: r \in R, s \in S\right.$ and $\left.g=(\operatorname{deg} s)^{-1}(\operatorname{deg} r)\right\}$. Let $M$ be a graded module over a $G$-graded ring $R$ and $S \subseteq h(R)$ be a multiplicatively closed subset of $R$. The module of fraction $S^{-1} M$ over a graded ring $S^{-1} R$ is a graded module which is called module of fractions, if $S^{-1} M=$ $g \in G \oplus\left(S^{-1} M\right)_{g}$ where $\left(S^{-1} M\right)_{g}=\{m / s: m \in M, s \in S$ and $g=$ $\left.(\operatorname{deg} s)^{-1}(\operatorname{deg} m)\right\}$. We write $h\left(S^{-1} R\right)=g \in G \cup\left(S^{-1} R\right)_{g}$ and $h\left(S^{-1} M\right)=$ $g \in G \cup\left(S^{-1} M\right)_{g}$. Consider the graded homomorphism $\eta: M \rightarrow S^{-1} M$ defined by $\eta(m)=m / 1$. For any graded submodule $N$ of $M$, the submodule of $S^{-1} M$ generated by $\eta(N)$ is denoted by $S^{-1} N$. Similar to non graded case, one can prove that $S^{-1} N=\left\{\beta \in S^{-1} M: \beta=m / s\right.$ for $m \in N$ and $\left.s \in S\right\}$ and that $S^{-1} N=S^{-1} M$ if and only if $S \cap\left(N:_{R} M\right)=\phi$. If $K$ is a graded submodule of $S^{-1} R$-module $S^{-1} M$, then $K \cap M$ will denote the graded submodule $\eta^{-1}(K)$ of $M$. Moreover, similar to the non graded case one can prove that $S^{-1}(K \cap M)=K$.

Let $R$ be a $G$-graded ring and $M$ a graded $R$-module.

A proper graded ideal $I$ of $R$ is said to be a graded maximal ideal of $R$ if $J$ is a graded ideal of $R$ such that $I \subseteq J \subseteq R$, then $I=J$ or $J=R$ (see[23].)

A proper graded ideal $I$ of $R$ is said to be a graded prime ideal if whenever $r s \in I$, we have $r \in I$ or $s \in I$, where $r, s \in h(R)$ (see [23].) The 
graded radical of $I$, denoted by $\operatorname{Gr}(I)$, is the set of all $x=\sum_{g \in G} x_{g} \in R$ such that for each $g \in G$ there exists $n_{g} \in Z^{+}$with $x^{n_{g}} \in I$. Note that, if $r$ is a homogeneous element, then $r \in \operatorname{Gr}(I)$ if and only if $r^{n} \in I$ for some $n \in N$ (see [23].) It is shown in [23, Proposition 2.5] that $\operatorname{Gr}(I)$ is the intersection of all graded prime ideals of $R$ containing $I$.

A proper graded ideal $P$ of $R$ is said to be a graded primary ideal if whenever $r, s \in h(R)$ with $r s \in P$, then either $r \in P$ or $s \in \operatorname{Gr}(P)$ (see [22].)

A proper graded submodule $N$ of $M$ is said to be a graded prime submodule if whenever $r \in h(R)$ and $m \in h(M)$ with $r m \in N$, then either $r \in\left(N:_{R} M\right)=\{r \in R: r M \subseteq N\}$ or $m \in N$ (see [7].) A proper graded submodule $N$ of a graded $R$-module $M$ is said to be a graded primary submodule if whenever $r \in h(R)$ and $m \in h(M)$ with $r m \in N$, then either $m \in N$ or $r \in \operatorname{Gr}\left(\left(N:_{R} M\right)\right)$ (see [11].)

The graded radical of a graded submodule $N$ of $M$, denoted by $\operatorname{Gr}_{M}(N)$, is defined to be the intersection of all graded prime submodules of $M$ containing $N$. If $N$ is not contained in any graded prime submodule of $M$, then $\operatorname{Gr}_{M}(N)=M$ (see [11].)

A graded $R$-module $M$ over $G$-graded ring $R$ is said to be a graded multiplication module (gr-multiplication module) if for every graded submodule $N$ of $M$ there exists a graded ideal $I$ of $R$ such that $N=I M$. It is clear that $M$ is $g r$-multiplication $R$ - module if and only if $N=\left(N:_{R} M\right) M$ for every graded submodule $N$ of $M$ (see [12].)

\section{Graded submodules which satisfy the gr-primeful property}

[5, Lemma 3.1]

The following Lemma is known (see [16, Lemma 1.2 and Lemma $2.7]$ ), we write it her for the sake of references.

Lemma 3.1. Let $R$ be a G-graded ring and $M$ a graded $R$-module. Then the following hold:

1. If $N$ is a graded submodule of $M$, then $\left(N:_{R} M\right)=\{r \in R: r M \subseteq$ $N\}$ is a graded ideal of $R$.

2. If $N$ is a graded submodule of $M, r \in h(R), x \in h(M)$ and $I$ is a graded ideal of $R$, then $R x, I N$ and $r N$ are graded submodules of $M$.

3. If $N$ and $K$ are graded submodules of $M$, then $N+K$ and $N \cap K$ are also graded submodules of $M$. 
4. If $\left\{N_{i}\right\}_{i \in I}$ is a collection of graded submodules of $M$, then $N=i \in I \cap$ $N_{i}$ is a graded submodule of $M$.

Definition 3.2. Let $N$ be a graded submodule of a graded $R$-module $M$. We say that $N$ satisfies the gr-primeful property if for each graded prime ideal $p$ of $R$ with $\left(N:_{R} M\right) \subseteq p$, there exists a graded prime submodule $P$ of $M$ containing $N$ such that $\left(P:_{R} M\right)=p$. A graded $R$-module $M$ is called gr-primeful, if either $M=0$ or the zero graded submodule of $M$ satisfies the gr-primeful property.

Theorem 3.3. Let $R$ be a $G$-graded ring and $M$ a graded $R$-module. If a graded submodule $N$ of $M$ satisfies the gr-primeful property, then $\operatorname{Gr}_{M}(N)$ satisfies the gr-primeful property.

Proof. Suppose that $p$ is a graded prime ideal of $R$ containing $\left(\operatorname{Gr}_{M}(N):_{R}\right.$ $M)$. Since $N$ satisfies the gr-primeful property and $\left(N:_{R} M\right) \subseteq\left(\operatorname{Gr}_{M}(N):_{R}\right.$ $M) \subseteq p$, there exists a graded prime submodule $P$ of $M$ containing $N$ such that $\left(P:_{R} M\right)=p$. It is clear that $\operatorname{Gr}_{M}(N) \subseteq P$. Thus $\operatorname{Gr}_{M}(N)$ satisfies the gr-primeful property.

Lemma 3.4. Let $R$ be a $G$-graded ring, $M$ a graded $R$-module and $N$ a graded submodule of $M$. Then $\operatorname{Gr}\left(\left(N:_{R} M\right)\right) \subseteq\left(G r_{M}(N):_{R} M\right)$.

Proof. Let $P$ be a graded prime submodule of $M$ containing $N$. By [7, Proposition 2.7(ii)], $\left(P:_{R} M\right)$ is a graded prime ideal of $R$. Since $N \subseteq P$, we get $\left(N:_{R} M\right) \subseteq\left(P:_{R} M\right)$. By [22, Proposition 1.2], we conclude that $\operatorname{Gr}\left(\left(N:_{R} M\right)\right) \subseteq\left(P:_{R} M\right)$. Thus $\operatorname{Gr}\left(\left(N:_{R} M\right)\right) M \subseteq\left(P:_{R} M\right) M \subseteq P$ and this that $\operatorname{Gr}\left(\left(N:_{R} M\right)\right) M \subseteq \operatorname{Gr}_{M}(N)$. Therefore $\operatorname{Gr}\left(\left(N:_{R} M\right)\right) \subseteq$ $\left(\operatorname{Gr}_{M}(N):_{R} M\right)$.

Theorem 3.5. Let $R$ be a $G$-graded ring and $M$ a graded $R$-module. If $N$ is a graded submodule of $M$ satisfying the gr-primeful property, then $\left(\operatorname{Gr}_{M}(N):_{R} M\right)=\operatorname{Gr}\left(\left(N:_{R} M\right)\right)$.

Proof. Let $p$ be a graded prime ideal of $R$ containing $\left(N:_{R} M\right)$. Since $N$ satisfies the gr-primeful property, there exists a graded prime submodule $P$ of $M$ containing $N$ such that $\left(P:_{R} M\right)=p$. This implies that $\left(\operatorname{Gr}_{M}(N):_{R}\right.$ $M) \subseteq\left(P:_{R} M\right) \subseteq p$ and hence $\left(\operatorname{Gr}_{M}(N):_{R} M\right) \subseteq \operatorname{Gr}\left(\left(N:_{R} M\right)\right)$. By Lemma 3.4, $\operatorname{Gr}\left(\left(N:_{R} M\right)\right) \subseteq\left(\operatorname{Gr}_{M}(N):_{R} M\right)$. Therefore $\left(\operatorname{Gr}_{M}(N):_{R}\right.$ $M)=\operatorname{Gr}\left(\left(N:_{R} M\right)\right)$. 
Let $R$ be a $G$-graded ring and $M, M^{\prime}$ graded $R$-modules. Let $\varphi$ : $M \rightarrow M^{\prime}$ be an $R$-module homomorphism. Then $\varphi$ is said to be a graded homomorphism if $\varphi\left(M_{g}\right) \subseteq M_{g}^{\prime}$ for all $g \in G$ (see [18].)

Theorem 3.6. Let $R$ be a $G$-graded ring and $M, M^{\prime}$ be two graded $R$ modules and $N^{\prime}$ a graded submodule of $M^{\prime}$. Let $\varphi: M \rightarrow M^{\prime}$ be a graded epimorphism. If $N^{\prime}$ satisfies the gr-primeful property, then so does $\varphi^{-1}$ ( $\left.N^{\prime}\right)$.

Proof. Let $p$ be a graded prime ideal of $R$ such that $\left(\varphi^{-1}\left(N^{\prime}\right):_{R} M\right) \subseteq$ $p$. We prove that $\left(N^{\prime}:_{R} M^{\prime}\right) \subseteq p$. Let $r \in\left(N^{\prime}:_{R} M^{\prime}\right) \cap h(R)$. Using the fact that $\varphi$ is a graded epimorphism, we have $r M^{\prime}=r \varphi(M)=\varphi(r M) \subseteq N^{\prime}$ and so $r M \subseteq \varphi^{-1}\left(N^{\prime}\right)$. Hence $r \in\left(\varphi^{-1}\left(N^{\prime}\right):_{R} M\right) \subseteq p$. Thus $\left(N^{\prime}:_{R} M^{\prime}\right) \subseteq p$. Since $N^{\prime}$ satisfies the gr-primeful property, there exists a graded prime submodule $P^{\prime}$ of $M^{\prime}$ containing $N^{\prime}$ such that $\left(P^{\prime}:_{R} M^{\prime}\right)=p$. By [10, Lemma 5.2(ii)], we have that $\varphi^{-1}\left(P^{\prime}\right)$ is a graded prime submodule of $M$ containing $\varphi^{-1}\left(N^{\prime}\right)$. It is easy to see $\left(\varphi^{-1}\left(P^{\prime}\right):_{R} M\right)=p$. Therefore $\varphi^{-1}($ $\left.N^{\prime}\right)$ satisfies the gr-primeful property.

Theorem 3.7. Let $R$ be a $G$-graded ring and $M, M^{\prime}$ be two graded $R$ modules. Let $\varphi: M \rightarrow M^{\prime}$ be a graded epimorphism and $N$ a graded submodule of $M$ containing Ker $\varphi$. If $N$ satisfies the gr-primeful property, then $\varphi(N)$ satisfies the gr-primeful property.

Proof. Let $p$ be a graded prime ideal of $R$ such that $\left(\varphi(N):_{R} M^{\prime}\right) \subseteq p$. We prove that $\left(N:_{R} M\right) \subseteq p$. Let $r \in\left(N:_{R} M\right) \cap h(R)$ so $r M \subseteq N$. Using the fact that $\varphi$ is a graded epimorphism, we have $r \varphi(M)=r M^{\prime} \subseteq \varphi(N)$. Hence $r \in\left(\varphi(N)::_{R} M^{\prime}\right) \subseteq p$. Thus $\left(N:_{R} M\right) \subseteq p$. Since $N$ satisfies the gr-primeful property, there exists a graded prime submodule $P$ of $M$ containing $N$ such that $\left(P:_{R} M\right)=p$. Since $\operatorname{Ker} \varphi \subseteq P$ by [10, Lemma 5.2(i)], we have $\varphi(P)$ is a graded prime submodule of $M^{\prime}$ containing $\varphi(N)$. It is easy to see $\left(\varphi(P):_{R} M^{\prime}\right)=p$. Therefore $\varphi(N)$ satisfies the gr-primeful property.

Theorem 3.8. Let $R$ be a $G$-graded ring, $M$ a graded $R$-module and $\left\{N_{i}\right.$ : $1 \leq i \leq n\}$ be a finite collection of graded submodules of $M$ satisfying the gr-primeful property. Then $\cap_{i=1}^{n} N_{i}$ satisfies the gr-primeful property. 
Proof. Let $p$ be a graded prime ideal of $R$ such that $\left(\cap_{i=1}^{n} N_{i}:_{R} M\right) \subseteq$ $p$. By [6, Lemma 2.6], we have $\left(\cap_{i=1}^{n} N_{i}:_{R} M\right)=\cap_{i=1}^{n}\left(N_{i}:_{R} M\right)$. Since $\cap_{i=1}^{n}\left(N_{i}: R M\right) \subseteq p$ by [22, Proposition 1.4], we have $\left(N_{j}:_{R} M\right) \subseteq p$ for some $j \in\{1,2, \ldots, n\}$. Since $N_{j}$ satisfies the gr-primeful property, there exists a graded prime submodule $P$ of $M$ containing $N_{j}$ with $\left(P:_{R} M\right)=p$ and so $\cap_{i=1}^{n} N_{i} \subseteq P$. Thus $\cap_{i=1}^{n} N_{i}$ satisfies the gr-primeful property.

\section{Gragded primary-like submodules}

Definition 4.1. Let $R$ be a $G$-graded ring and $M$ a graded $R$-module. A proper graded submodule $N$ of $M$ is said to be a graded primary-like submodule if whenever $r \in h(R)$ and $m \in h(M)$ with $r m \in N$, then either $r \in(N: R M)$ or $m \in G_{M}(N)$.

Theorem 4.2. Let $R$ be a $G$-graded ring and $M$ a graded $R$-module. If $N$ is a graded primary-like submodule of $M$ satisfying the gr-primeful property, then $\left(N:_{R} M\right)$ is a graded primary ideal of $R$.

Proof. Suppose that $r s \in\left(N:_{R} M\right)$ and $s \notin\left(N:_{R} M\right)$ for some $r, s \in h(R)$. We show that $r \in\left(\operatorname{Gr}(N):_{R} M\right)$. Let $m=\sum_{g \in G} m_{g} \in M$. Hence $\sum_{g \in G} r s m_{g} \in N$. Since $N$ is a graded primary-like submodule of $M$, $r s m_{g} \in N$ and $s \notin\left(N:_{R} M\right)$ for all $g \in G$, we conclude that $r m_{g} \in \operatorname{Gr}_{M}(N)$ for all $g \in G$. Hence $r m \in \operatorname{Gr}_{M}(N)$. This shows that $r \in(\operatorname{Gr}(N): M)$. Since $N$ satisfies the gr-primeful property, by Theorem 3.5, we have $r \in$ $\left(\operatorname{Gr}(N):_{R} M\right)=\operatorname{Gr}\left(\left(N:_{R} M\right)\right)$. Therefore $\left(N:_{R} M\right)$ is a graded primary ideal of $R$.

By [22, Lemma 1.8] and Theorem 4.2, we have the following Corollary.

Corollary 4.3. Let $R$ be a $G$-graded ring and $M$ a graded $R$-module. If $N$ is a graded primary-like submodule of $M$ satisfying the gr-primeful property, then $p=\operatorname{Gr}\left(\left(N:_{R} M\right)\right)$ is a graded prime ideal of $R$ and we say that $N$ is a graded p-primary-like submodule of $M$.

Theorem 4.4. Let $R$ be a $G$-graded ring, $M$ a faithful graded multiplication $R$-module and $Q$ a graded primary deal of $R$. If $r m \in Q M$ for $r \in h(R), m \in h(M)$, then $r \in Q$ or $m \in G r_{M}(Q M)$.

Proof. The proof is similar to the proof of [13, Theorem 2.2] and [21, Lemma 2], so we omit it. 
Corollary 4.5. Let $R$ be a $G$-graded ring, $M$ a faithful graded multiplication $R$-module and $Q$ a graded primary ideal of $R$ such that $M=Q M$, then $Q M$ is a graded primary-like submodule of $M$.

Proof. Let $r m \in Q M$ where $r \in h(R)$ and $m \in h(M)$. So $r \in Q \subseteq$ $\left(Q M:_{R} M\right)$ or $m \in \operatorname{Gr}_{M}(Q M)$ by Theorem 4.4. Therefore, $Q M$ is a graded primary-like submodule of $M$.

Theorem 4.6. Let $R$ be a $G$-graded ring, $M$ a graded multiplication $R$ module and $N$ a proper graded submodule of $M$. If $N$ satisfies the grprimeful property, then the following statements are equivalent.

1. $N$ is a graded primary-like submodule of $M$;

2. $\left(N:_{R} M\right)$ is a graded primary ideal of $R$;

3. $N=Q M$ for some graded primary ideal $Q$ of $R$ with $\operatorname{Ann}(M)=$ $(0: R M) \subseteq Q$

4. $N$ is graded primary submodule of $M$.

Proof. $\quad(i) \Rightarrow(i i)$ By Theorem 4.2. (ii) $\Leftrightarrow($ iii $) \Leftrightarrow(i v)$ By [13, Corollary 2.5]. $($ iii $) \Rightarrow(i)$ Let $Q$ be a graded primary ideal of $R$ such that $N=Q M$ and $\operatorname{Ann}(M) \subseteq Q$. Then $M$ is a faithful graded $R / A n n(M)$-module. The result follows from Corollary 4.5.

Corollary 4.7. Let $R$ be a $G$-graded ring and $M$ a graded multiplication $R$-module. If $N$ is a graded primary-like submodule of $M$ satisfying the gr-primeful property, then $\operatorname{Gr}(N)$ is a graded prime submodule of $M$.

Proof. Let $N$ be a graded primary-like submodule of $M$. By Theorem 4.6, $N=Q M$ for some graded primary ideal $Q$ of $R$ containing $\operatorname{Ann}(M)$. Since $M$ is a graded multiplication module by [21, Theorem 9], $\operatorname{Gr}_{M}(Q M)=\operatorname{Gr}(Q) M$. By [22, Lemma 1.8] and [11, Proposition 2.6(ii)], we conclude that $\operatorname{Gr}(Q) M$ is a graded prime submodule. Therefore $\operatorname{Gr}(N)$ is a graded prime submodule of $M$.

Theorem 4.8. Let $R$ be a $G$-graded ring, $M$ a non-zero graded $R$-module and $N$ a proper graded submodule of $M$. Then the following statements are equivalent. 
1. $N$ is a graded primary-like submodule of $M$;

2. $\left(N:_{R} M\right)=\left(N:_{R} U\right)$ for every graded submodule $U$ of $M$ such that $\operatorname{Gr}_{M}(N) U$;

Proof. $\quad(i) \Rightarrow(i i)$ Assume that $N$ is a graded primary-like submodule of $M$ and let $U$ be any graded submodule of $M$ such that $\operatorname{Gr}_{M}(N) U$. We show that $\left(N:_{R} U\right)=\left(N:_{R} M\right)$. Clearly $\left(N:_{R} M\right) \subseteq\left(N:_{R} U\right)$. Let $r=\sum_{g \in G} r_{g} \in\left(N:_{R} U\right)$. Hence for any $g \in G$, we have $r_{g} U \subseteq N$. Since $\operatorname{Gr}_{M}(N) U$, there exists $u_{h} \in h(U)-\operatorname{Gr}_{M}(N)$. Since $N$ is a graded primary-like submodule of $M, r_{g} u_{h} \in N$ and $u_{h} \notin \operatorname{Gr}_{M}(N)$ for all $g \in G$, we conclude that $r_{g} \in\left(N:_{R} M\right)$ for all $g \in G$. Hence $r \in\left(N:_{R} M\right)$. Therefore $\left(N:_{R} U\right)=\left(N:_{R} M\right)$.

$($ ii $) \Rightarrow(i)$ : Suppose that $r_{g} m_{h} \in N$ and $m_{h} \notin \operatorname{Gr}_{M}(N)$ for some $r_{g} \in h(R)$ and $m_{h} \in h(M)$. By Lemma 3.1, $U=\operatorname{Gr}_{M}(N)+R m_{h}$ is a graded submodule of $M$. Since $m_{h} \notin \operatorname{Gr}_{M}(N)$, we have $\operatorname{Gr}_{M}(N) U \subseteq M$. By (ii), $\left(N:_{R} U\right)=\left(N:_{R} M\right)$. Since $r_{g} m_{h} \in N$, we have $r_{g}\left(N+R m_{h}\right)=$ $r_{g} N+R r_{g} m \subseteq N$. This yields that $r_{g} \in\left(N:_{R} N+R m_{h}\right)=\left(N:_{R} M\right)$. Therefore $N$ is a graded primary-like submodule of $M$.

Theorem 4.9. Let $R$ be a $G$-graded ring and $M, M^{\prime}$ be two graded $R$ modules and $N^{\prime}$ a graded submodule of $M^{\prime}$. Let $\varphi: M \rightarrow M^{\prime}$ be a graded epimorphism. If $N^{\prime}$ is a graded primary-like submodule of $M^{\prime}$, then $\varphi^{-1}$ ( $N^{\prime}$ ) is a graded primary-like submodule of $M$.

Proof. Suppose that $r_{g} m_{h} \in \varphi^{-1}\left(N^{\prime}\right)$ and $r_{g} \notin\left(\varphi^{-1}\left(N^{\prime}\right):_{R} M\right)$ for some $r_{g} \in h(R)$ and $m_{h} \in h(M)$. Hence $\varphi\left(r_{g} m_{h}\right)=r_{g} \varphi\left(m_{h}\right) \in N^{\prime}$. Since $r_{g} \notin\left(\varphi^{-1}\left(N^{\prime}\right):_{R} M\right)$, we get $r_{g} \notin\left(N^{\prime}:_{R} M^{\prime}\right)$. Since $N^{\prime}$ is a graded primary-like submodule of $M^{\prime}, r_{g} \varphi\left(m_{h}\right) \in N^{\prime}$ and $r_{g} \notin\left(N^{\prime}:_{R} M^{\prime}\right)$, we have $\varphi\left(m_{h}\right) \in \operatorname{Gr}_{M^{\prime}}\left(N^{\prime}\right)$ it follows that $m_{h} \in \varphi^{-1}\left(\operatorname{Gr}_{M^{\prime}}\left(N^{\prime}\right)\right)$. By [10, Theorem 5.3(i)], we have $\varphi^{-1}\left(\operatorname{Gr}_{M^{\prime}}\left(N^{\prime}\right)\right)=\operatorname{Gr}_{M}\left(\varphi^{-1}\left(N^{\prime}\right)\right)$. Hence $m_{h} \in \operatorname{Gr}_{M}\left(\varphi^{-1}\left(N^{\prime}\right)\right)$. Therefore $\varphi^{-1}\left(N^{\prime}\right)$ is a graded primary-like submodule of $M$.

Lemma 4.10. Let $R$ be a $G$-graded ring and $M, M^{\prime}$ be two graded $R$ -modules. Let $\varphi: M \rightarrow M^{\prime}$ be a graded epimorphism and $N$ a graded submodule of $M$ containing $\operatorname{Ker} \varphi$. Then $\varphi\left(G_{M}(N)\right)=G_{M^{\prime}}(\varphi(N))$. 
Proof. The proof is similar to the proof of [10, Theorem 5.3(ii)], so we omit it.

Theorem 4.11. Let $R$ be a $G$-graded ring and $M, M^{\prime}$ be two graded $R$-modules. Let $\varphi: M \rightarrow M^{\prime}$ be a graded epimorphism and $N$ a graded submodule of $M$ containing $\operatorname{Ker} \varphi$. If $N$ is a graded primary-like submodule of $M$, then $\varphi(N)$ is a graded primary-like submodule of $M^{\prime}$.

Proof. Suppose that $r m^{\prime} \in \varphi(N)$ and $r \notin\left(\varphi(N):_{R} M^{\prime}\right)$ for some $r \in h(R)$ and $m^{\prime} \in h\left(M^{\prime}\right)$. It is easy to see $r \notin(N: R M)$. Since $\varphi$ is a graded epimorphism, there exists $m \in h(M)$ such that $\varphi(m)=m^{\prime}$ and hence $r \varphi(m)=\varphi(r m) \in \varphi(N)$. Thus there exists $n \in h(N)$ such that $\varphi(r m)=\varphi(n)$ this implies $r m-n \in \operatorname{Ker} \varphi \subseteq N$. This yields that $r m \in N$. Since $N$ is a graded primary-like submodule of $M, r m \in N$ and $r \notin\left(N:_{R}\right.$ $M)$ ), we conclude that $m \in G_{M}(N)$. Hence $\varphi(m)=m^{\prime} \in \varphi\left(G_{M}(N)\right)$. By Lemma 4.10, we have $m^{\prime} \in \varphi\left(G_{M}(N)\right)=G_{M^{\prime}}(\varphi(N))$. Therefore $\varphi(N)$ is a graded primary-like submodule of $M^{\prime}$.

Lemma 4.12. Let $R$ be a $G$-graded ring and $M$ a graded $R$-module. Let $S \subseteq h(R)$ be a multiplicatively closed subset of $R$ and $N$ a graded primarylike submodule of $M$ satisfying the gr-primeful property such that $S \cap$ $\operatorname{Gr}\left(\left(N:_{R} M\right)\right)=\emptyset$. Then $S^{-1}\left(N:_{R} M\right)=\left(S^{-1} N:_{S^{-1} R} S^{-1} M\right)$.

Proof. Let $\frac{r}{s} \in\left(S^{-1} N:_{S^{-1} R} S^{-1} M\right)$ for some $\frac{r}{s} \in h\left(S^{-1} R\right)$. Since $N$ satisfies the gr-primeful property, there exists $m_{g} \in h(M) \backslash \mathrm{Gr}_{M}(N)$. Thus $\frac{r}{s} \frac{m_{g}}{1}=\frac{r m_{g}}{-s} \in S^{-1} N$. Hence there exists $u \in S$ such that $u_{r m} \in N$. Since $N$ is a graded primary-like submodule, $u r m_{g} \in N$ and $m_{g} \notin \operatorname{Gr}_{M}(N)$, we conclude that ur $\in\left(N:_{R} M\right)$. By Theorem $4.2,\left(N:_{R} M\right)$ is a graded primary ideal of $R$. Since $S \cap \operatorname{Gr}\left(\left(N:_{R} M\right)\right)=\emptyset$ and $u r \in\left(N:_{R} M\right)$, we have $r \in\left(N:_{R} M\right)$ and hence $\frac{r}{s} \in S^{-1}\left(N:_{R} M\right)$. So $\left(S^{-1} N:_{S^{-1} R}\right.$ $\left.S^{-1} M\right) \subseteq S^{-1}\left(N:_{R} M\right)$. It is clear that $S^{-1}\left(N:_{R} M\right) \subseteq\left(S^{-1} N:_{S^{-1} R}\right.$ $\left.S^{-1} M\right)$. Thus $S^{-1}\left(N:_{R} M\right)=\left(S^{-1} N:_{S^{-1} R} S^{-1} M\right)$.

The following results study the behavior of graded primary-like submodules under localization.

Theorem 4.13. Let $R$ be a $G$-graded ring and $M$ a graded $R$-module. Let $S \subseteq h(R)$ be a multiplicatively closed subset of $R$ and $N$ a graded primary-like submodule of $M$ satisfying the gr-primeful property such that $S \cap G r\left(\left(N:_{R} M\right)\right)=\emptyset$. Then $S^{-1} N$ is a graded primary-like submodule of $S^{-1} R$-module $S^{-1} M$ satisfying the gr-primeful property. 
Proof. It is easy to see that $\frac{m}{1} \in S^{-1} M \backslash S^{-1} N$ for each $m \in M \backslash N$ and hence $S^{-1} N=S^{-1} M$. Suppose that $\frac{r}{s} \frac{m}{l} \in S^{-1} N$ and $\frac{r}{s} \notin\left(S^{-1} N:_{S^{-1} R}\right.$ $\left.S^{-1} M\right)$ for some $\frac{r}{s} \in h\left(S^{-1} R\right)$ and for some $\frac{m}{l} \in h\left(S^{-1} M\right)$. Since $S^{-1}\left(\left(N:_{R}\right.\right.$ $M)) \subseteq\left(S^{-1} N:_{S^{-1} R} S^{-1} M\right)$, we have $r \notin\left(N:_{R} M\right)$. Thus there exists $k \in S$ such that $\mathrm{krm} \in N$. By Theorem 4.2, $\left(N:_{R} M\right)$ is a graded primary ideal of $R$. Since $S \cap \operatorname{Gr}\left(\left(N:_{R} M\right)\right)=\emptyset$ and $r \notin\left(N:_{R} M\right)$, we conclude that $k r \notin\left(N:_{R} M\right)$. So $N$ a is graded primary-like submodule of $M$ gives $m \in \operatorname{Gr}_{M}(N)$. Hence $\frac{m}{l} \in S^{-1}\left(\operatorname{Gr}_{M}(N)\right) \subseteq \operatorname{Gr}_{M}\left(S^{-1} N\right)$. Therefore $S^{-1} N$ is a graded primary-like submodule of $S^{-1} M$.

Now we prove that $S^{-1} N$ satisfies the gr-primeful property. Let $S^{-1} p$ be a graded prime ideal of $S^{-1} R$ containing $\left(S^{-1} N:_{S^{-1} R} S^{-1} M\right)$. Hence $p \cap S=\emptyset$ and $S^{-1}\left(\left(N:_{R} M\right)\right) \subseteq\left(S^{-1} N: S^{-1} R S^{-1} M\right) \subseteq S^{-1} p$. Then $S^{-1}\left(\left(N:_{R} M\right)\right) \subseteq S^{-1} p$ and so easily follows that $\left(N:_{R} M\right) \subseteq p$. Since $N$ satisfies the gr-primeful property, there exists a graded prime submodule $P$ of $M$ containing $N$ such that $\left(P:_{R} M\right)=p$. Since $p \cap S=\emptyset, S^{-1} P$ is a graded prime submodule of $S^{-1} M$ containing $S^{-1} N$ and $\left(S^{-1} P:_{S^{-1} R}\right.$ $\left.S^{-1} M\right)=S^{-1} p$. Therefore $S^{-1} N$ satisfies the gr-primeful property.

\section{References}

[1] K. Al-Zoubi, "The graded primary radical of a graded submodules", Analele ştiințifice ale Universităţi "Al.I. Cuza" din Iaşi. Matematică (Online), vol. 1, no. 2, pp. 395-402, 2016. [On line]. Available: https:/ / bit.ly/ 36f5e3s

[2] K. Al-Zoubi and R. Abu-Dawwas, "On graded quasi-prime submodules", Kyungpook mathematical journal, vol. 55, no. 2, pp. 259-266, 2015. doi: 10.5666/ KMJ.2015.55.2.259

[3] K. Al-Zoubi, R. Abu-Dawwas and I. Al-Ayyoub, "Graded semiprime submodules and graded semi-radical of graded submodules in graded modules", Ricerche di matematica, vol. 66, no. 2, pp. 449-455, 2017. doi: 10.1007/ s11587-016-0312-x

[4] K. Al-Zoubi and M. Al-Dolat, "On graded classical primary submodules", Advances in pure and applied mathematics, vol. 7, no. 2, pp. 93-96, 2016. doi: 10.1515/ apam-2015-0021

[5] K. Al-Zoubi, M. Jaradat and R. Abu-Dawwas, "On graded classical prime and graded prime submodules", Bulletin of the Iranian Mathematical Society, vol. 41, no. 1, pp. 217-225, 2015. [On line]. Available: https:/ / bit.ly/ 2TB9K9Y

[6] K. Al-Zoubi and F. Qarqaz, "An intersection condition for graded prime submodules in Gr-multiplication modules", Mathematicals reports, vol. 20, no. 3, pp. 329-336, 2018. [On line]. Available: https:// bitly/ 2TL9KUC 
[7] S. E. Atani, "On graded prime submodules", Chiang mai journal of science, vol. 33, no. 1, pp. 3-7, 2006. [On line]. Available: https:/ / bit.ly/ 3wmHTYr

[8] S. E. Atani and R. E. Atani, "Graded multiplication modules and the graded ideal $\theta_{\mathrm{g}}(\mathrm{M})$ ", Turkish journal of mathematics, vol. 35, no. 1, pp. 1-9, 2009. doi: 10.3906/ mat-0901-22

[9] S. E. Atani and F. Farzalipour, "Notes on the graded prime submodules", International mathematical forum, vol. 1, no. 38, pp. 1871-1880, 2006. doi.org: 10.12988/ imf.2006.06162

[10] S. E. Atani and F. E. K. Saraei, "Graded modules which satisfy the Gr-radical formula", Thai journal of mathematics, vol. 8, no. 1, pp. 161-170, 2010. [On line]. Available: https:/ / bit.ly/ 3e8mZWL

[11] S. E. Atani and F. Farzalipour, "On graded secondary modules", Turkish journal of mathematics, vol. 31, no. 4, pp. 371-378, 2007. [On line]. Available: https:/ / bit.ly/ 3yt7wbh

[12] J. Escoriza and B. Torrecillas, "Multiplication objects in commutative Grothendieck categories", Communications in algebra, vol. 26, no. 6, pp. 1867-1883, 1998. doi: 10.1080/00927879808826244

[13] P. Ghiasvand and F. Farzalipour, "On graded primary submodules of graded multiplication modules", International journal of algebra, vol. 4, no. 9, pp. 429-434, 2010. [On line]. Available: https:/ / bit.ly/ 3wgHirb

[14] R. Hazrat, Graded rings and graded Grothendieck groups, Cambridge: Cambridge University Press, 2016. doi: 10.1017/ CBO9781316717134

[15] C. P. Lu, "A module whose prime spectrum has the surjective natural map", Houston journal of mathematics, vol. 33, no. 1, pp. 125-143, 2007. [On line]. Available: https:/ / bit.ly/ 36f7F68

[16] S. C. Lee and R. Varmazyar, "Semiprime submodules of graded multiplication modules", Journal of the Korean Mathematical Society, vol. 49, no. 2, pp. 435-447, 2012. doi: 10.4134/JKMS.2012.49.2.435

[17] H. F. Moghimi and F. Rashedi, "Primary-like submodules satisfying the primeful property", Transactions on algebra and its applications, vol. 1, pp. 43-54, 2015.

[18] C. Nastasescu and F. Oystaeyen, Graded and filtered rings and modules. Berlin: Springer, 1979. doi: 10.1007/ BFb0067331

[19] C. Nastasescu, F. Oystaeyen, Graded ring theory. Amsterdam: North Holland, 1982. 
[20] C. Nastasescu and F. Oystaeyen, Methods of graded rings. Berlin: Springer, 2004. doi: 10.1007/ b94904

[21] K. H. Oral, U. Tekir and A.G. Agargun, "On Graded prime and primary submodules", Turkish journal of mathematics, vol. 35, no. 2, pp. 159-167, 2011. doi: 10.3906/ MAT-0904-11

[22] M. Refai and K. Al-Zoubi, "On graded primary ideals", Turkish journal of mathematics, vol. 28, no. 3, pp. 217-229, 2004. [On line]. Available: https:/ / bit.ly/ 3hMUvTs

[23] M. Refai, M. Hailat and S. Obiedat, "Graded radicals on graded prime spectra", Far East journal of mathematical sciences, vol. 1, no. 1, pp. 59-73, 2000.

[24] H. A. Tavallaee and M. Zolfaghari, "Graded weakly semiprime submodules of graded multiplication modules", Lobachevskii journal of mathematics, vol. 34, no. 1, pp. 61-67, 2013. doi: 10.1134/ S1995080213010113 